\title{
Assessment of Hand Grip Strength of Orthopedically Challenged Persons Affected with Upper Extremity
}

\author{
Prakash Chandra Dhara*, Sujaya De, Amitava Pal , Piyali Sengupta and Sudeshna Roy \\ Ergonomics and Sports Physiology Division, Department of Human Physiology with \\ Community Health, Vidyasagar University, Medinipur 721 102, West Bengal, India \\ *E mail: prakashdhara@rediffmail.com,prakashcdhara@gmail.com
}

KEYWORDS Physically Challenged. Muscle Strength. Body Dimension

\begin{abstract}
The present investigation was aimed to assess the extent of variation in grip strength in relation to the sex and type of deformity among the orthopedically challenged persons affected with upper extremity and to compare the grip strength with that of control (normal) persons. For this purpose 213 subjects having the age range 20-60 years were selected at random. Among them 109 subjects were orthopedically challenged and 104 subjects were normal, who were treated as control subjects. Orthopedically challenged subjects were taken from three categories, viz., stroke patient, accidental patient and arthritis patient. Hand grip strength was measured by using Lafayette Hand Grip Dynamometer. Some anthropometric measures of the subjects of both groups were taken. The result showed that the hand grip strength of orthopedically challenged subjects was significantly lesser $(p<0.001)$ than that of the control subjects in both hands. The male subjects had significantly higher hand grip strength than that of the female subjects in both groups. The mean values of different anthropometric measures also showed the same results. The grip strength was found to be significantly correlated with a few anthropometric measures of the subjects. The results of the ANOVA showed that the grip strength of different categories of orthopedically challenged persons had significant variations ( $p<0.01$ ) only in case of left hand of female subjects. It can be concluded that the lower value of hand grip strength of the orthopedically challenged persons may be considered during designing control panel, switches and other hand arm operated devices.
\end{abstract}

\section{INTRODUCTION}

Hand grip strength is an easily obtainable measure of physical health and muscle function. Reliable and valid evaluation of hand strength is very important in determining the effectiveness of various surgical or treatment procedure. During grip exertion the most exposed areas are the metacarpal regions (Muralidhar and Bishu 2000). After acquiring a disability, the amount of physical activity is found to decrease rapidly, which leads to a loss of muscle mass and diminished level of strength (Janssen et al. 1994; Rimmer 2001). Many people with disabilities fall into a sedentary lifestyle, which usually leads to additional medical problems, such as heart and lung disease, pressure sores, obesity, osteo-

Corresponding Author:

Prakash Chandra Dhara

Ergonomics and Sports Physiology Division

Department of Human physiology with Community

Health, Vidyasagar University, Medinipore 721102

West Bengal, India

Telephone: 91-03222-276554 ext - 450,

Fax: 91-03222- 275329

Mobile: 09433226695

E-mail: prakashdhara@rediffmail.com/

prakashcdhara@gmail.com porosis, joint pain, and arthritis (Janssen et al. 1994; Rimmer 2001). With increasing age, muscle strength decreases and may eventually reach a level at which weakness starts to restrict the ability to perform usual activities (Young 1986; Porter et al. 1995). Mathiowetz et al. (1985a) studied the effect of elbow position on grip strength and found it to be higher with the elbow flexed at $90^{\circ}$. The American Society of Hand Therapists (Fess et al. 1981), suggesting a standardized arm positioning for hand strength tests, concluded that the position of upper extremity might influence measurements and recommended that the patient should be seated with his shoulder adducted and neutrally rotated, elbow flexed at $90^{\circ}$ and the forearm and wrist in neutral position. The grip strength test measures the muscular strength of our upper extremities. Grip strength is often different for each hand and the preferred hand was usually the strongest. There is a strong correlation between grip strength and overall upper body strength. A number of studies have shown that hand grip strength is both a highly sexually dimorphic and a lateralized anthropometric measurement (Mathiowetz et al. 1985b; Petersen et al. 1989; Kamarul et al. 2006). Strength of the grip can be used as an index of 
recovery of arm function. Boissy et al. (1999) suggest that the paretic maximal grip strength, normalized with the maximal grip strength on the non affected side, appears to be a valuable outcome measure of upper extremity function in chronic stroke subjects. Mercierand et al. (2004) noted that strength is related to the function of the paretic upper limb. Any of the dynamometrically measured actions are adequate for characterizing strength of either side after stroke, simplicity of handgrip dynamometry upholds its utilization. Asymmetrical weakness is often found in people who have cerebral palsy or encounter a stroke (Rimmer 2001). If there is weakness to one side of the body, the professional team (therapists, trainers, caregivers, and/or exercise physiologist) may want to make sure that active assistance is used when working with the affected side during resistance training (Rimmer 2001). Sunderland et al. (1989) observed that improvement in grip strength scores in a group of 38 acute stroke patients closely paralleled improvements on more complex motor tasks. This finding suggests that grip strength may be a valuable marker for recovery of arm function in acute stroke patients. Zhang et al. (2002) found that symptomatic hand osteoarthritis is a common disease among an elderly population. Further, subjects with symptomatic hand osteoarthritis have weaker grip strength and often have impairment in hand functions that require either precise pincer grip or power grip. Grip strength has a specific role in ergonomics especially for hand tool using. In ergonomics a hand tool must be designed such a way that provide a better fit for the user. Fit it terms of comfort and efficiency of use, is particularly important for tools used for long term projects for occupational activities. It also reduces the risk potential injuries associated with using of hand tool. Strength depends upon different factors like age, sex, arm position, wrist position and muscle mass etc. In general maximum strength reaches peak between the ages of 25-35 years, shows a gradual increase and than accelerated decline with advancing age (Kallman et al. 1990). Female grip strength was $61.8 \%$ of the male value for the $60-69$ years age group and decreased to $46.7 \%$ for age 90+ (Chaparro et al. 2000).

In the present investigation an attempt has been made to find out the degree of variation in grip strength between orthopedically challenged and normal persons as well as to assess the difference in grip strength among different categories of orthopedically challenged subjects.

\section{METHODOLOGY}

Site and Subjects: The study was carried out in National Institute of Orthopedically Handicapped in Kolkata, and in Midnapore Rehabilitation Centre for Children (MRCC) located in Paschim Midnapore District, West Bengal for orthopedically challenged subjects. For the controlled subjects study was conducted in municipal areas of Midnapore town and different villages of Midnapore Sadar subdivision. For this purpose 213 subjects having the age range 20-60 years were selected at random. Among them 109 subjects were orthopedically challenged and 104 subjects were normal, who were treated as control group. Orthopedically challenged subjects had upper extremity disorder and they were divided into three categories, viz., stroke patient, accidental patient, and arthritis patient. Both orthopedically challenged and control subjects were further divided into male and female groups. All the subjects volunteered for the study.

Measurement of Hand Grip Strength: The static grip strength measurement was performed by having the subjects' maximal grip by using hand grip dynamometer (Lafayette, USA). This hand grip dynamometer features a dual pointer system to retain the minimum effort. The testing range on a dual scale was (0-100) $\mathrm{kg} /(0-220) \mathrm{lb}$. Before taking measurements the subjects were requested to stand in a comfortable position. The subject was asked to squeeze the dynamometer as hard as possible without moving the rest of the body. Thus the final grip strength measured for both hand and reading was taken from the dynamometer scale, when the pointer no longer moved. The test was performed when the upper arm was hanging normally beside the body with fore arm forming an angle of $90^{\circ}$ at elbow.

Anthropometric Measurements: These were taken (height, weight, total arm length, upper arm length, lower arm length, palm length, fist girth, and hand grip circumference) in standardized condition and using proper landmarks after Weiner and Lourie (1969), Ermakova et al. (1985) Sodhi (1991).

\section{RESULTS}

A comparison of hand grip strength was made between the subjects of orthopedically challenged and control groups (Table 1). Results showed that the hand grip strength was 
Table 1: Comparison of hand grip strength $(\mathrm{kg})(\mathrm{Mean} \pm \mathrm{SD})$ between orthopedically challenged and control subjects

$(\mathrm{CM}=$ Control Male, $\mathrm{CF}=$ Control Female, $\mathrm{OCM}=$ Orthopedically challenged Male, $\mathrm{OCF}=$ Orthopedically challenged Female, RH=Right Hand, LH=Left Hand)

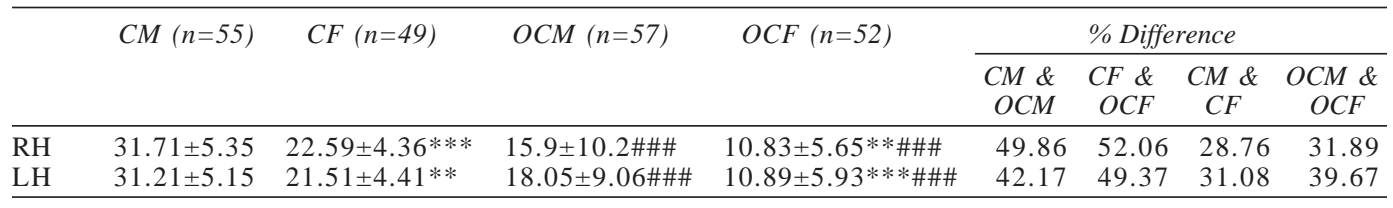

w.r.t male subjects $* * * \mathrm{p}<0.001 * * \mathrm{p}<0.01$

w.r.t control subjects \#\#\# p<0.001

significantly higher in male subjects than that of the female subjects in both categories of subjects. There was also a significant difference $(\mathrm{p}<0.001)$ in grip strength between the orthopedically challenged and control subjects in both the sexes. There was a remarkable variation in hand grip strength between orthopedically challenged subject and control subjects. The grip strength of control male subjects was $49.86 \%$ higher in right hand and $42.17 \%$ higher in left hand than that of the male orthopedically challenged subjects; the values were $52.06 \%$ and $49.37 \%$ higher in right hand and left hand respectively in control female subjects than that of the female orthopedically challenged subjects.

Anthropometric parameters viz. height, weight, arm length, palm length, hand grip circumference and fist girth were compared between the group and within the group (Table $2)$. This result showed that different anthropometric dimensions were significantly higher in male in comparison to the female subjects among the orthopedically challenged and control subjects. In case of male subjects, there was a significant difference in different anthropometric measures, viz., weight, total arm length, fist girth and hand grip circumference between control and orthopedically challenged subjects. On the other hand, anthropometric measures, viz., upper arm length, fist girth and hand grip circumference had significant difference between orthopedically challenged and control female subjects.

Correlation coefficient (r) between hand grip strength and different anthropometric variables of both male and female control and orthopedically challenged subjects has been presented in Table 3. In male and female orthopedically challenged subjects, the correlation coefficient between hand grip strength and other anthropometric variables, viz., hand grip circumference, palm length, arm length and fist girth represented poor correlation in most of the cases. However, in female orthopedically challenged subjects, the grip strength was significantly correlated with

Table 2: Comparison of anthropometric parameter $(M e a n \pm S D)$ between orthopedically challenged and control subjects

\begin{tabular}{|c|c|c|c|c|c|}
\hline & & \multicolumn{2}{|c|}{ Control } & \multicolumn{2}{|c|}{ Orthopedically challenged } \\
\hline & & Male & Female & Male & Female \\
\hline \multicolumn{2}{|l|}{ Height $(\mathrm{cm})$} & $165.17 \pm 5.4$ & $153.32 \pm 5.48 * * *$ & $166.32 \pm 6.34$ & $150.55 \pm 12.19 * * *$ \\
\hline \multicolumn{2}{|l|}{ Weight $(\mathrm{kg})$} & $57.4 \pm 6.26$ & $53.08 \pm 7.32 * * *$ & $61.75 \pm 8.54 \# \# \#$ & $56.96 \pm 16.28 * * *$ \\
\hline \multirow[t]{2}{*}{ Total arm length $(\mathrm{cm})$} & Right Hand & $54.35 \pm 3.92$ & $50.08 \pm 2.75 * * *$ & $56.34 \pm 4.67 \#$ & $51.34 \pm 4.15 * * *$ \\
\hline & Left Hand & $54.41 \pm 3.9$ & $49.38 \pm 2.71 * * *$ & $55.85 \pm 4.65$ & $51.6 \pm 4.15 * * *$ \\
\hline \multirow[t]{2}{*}{ Upper arm length $(\mathrm{cm})$} & Right Hand & $29.87 \pm 1.91$ & $28.51 \pm 3.42 * * *$ & $30.35 \pm 2.13$ & $27.53 \pm 1.95 * * *$ \\
\hline & Left Hand & $30.06 \pm 2.65$ & $28.46 \pm 1.66 *$ & $30.52 \pm 3.12$ & $27.62 \pm 1.85 * * * \#$ \\
\hline \multirow[t]{2}{*}{ Lower arm length $(\mathrm{cm})$} & Right Hand & $24.16 \pm 3.35$ & $22.06 \pm 2.31 * * *$ & $24.86 \pm 2.46$ & $22.84 \pm 2.32 * * *$ \\
\hline & Left Hand & $24.19 \pm 3.3$ & $22.12 \pm 2.31 * * *$ & $24.79 \pm 2.39$ & $22.74 \pm 2.08 * * *$ \\
\hline \multirow[t]{2}{*}{ Palm length (cm) } & Right Hand & $10.18 \pm 0.67$ & $9.3 \pm 0.64 * * *$ & $10.66 \pm 2.31$ & $9.17 \pm 0.8 * * *$ \\
\hline & Left Hand & $10.14 \pm 0.69$ & $9.24 \pm 0.59 * * *$ & $10.72 \pm 2.39$ & $9.3 \pm 1.69 * * *$ \\
\hline \multirow[t]{2}{*}{ Fist girth $(\mathrm{cm})$} & Right Hand & $24.9 \pm 2.04$ & $22.59 \pm 2.12 * * *$ & $26.56 \pm 2.76 \# \# \#$ & $23.86 \pm 2.89 * * * \#$ \\
\hline & Left Hand & $26.88 \pm 2.16$ & $22.88 \pm 2.02 * * *$ & $26.53 \pm 2.68 \# \# \#$ & $23.71 \pm 2.89 * * *$ \\
\hline \multirow{2}{*}{$\begin{array}{l}\text { Hand grip circumference } \\
(\mathrm{cm})\end{array}$} & Right Hand & $18.65 \pm 2.44$ & $16.97 \pm 2.92 *$ & $15.68 \pm 3.64 \# \# \#$ & $12.64 \pm 3.04 * * * \# \# \#$ \\
\hline & Left Hand & $18.63 \pm 2.66$ & $16.59 \pm 3.13 * * *$ & $15.71 \pm 3.58 \# \# \#$ & $12.77 \pm 2.64 * * * \# \# \#$ \\
\hline
\end{tabular}

w.r.t male subjects $* * * \mathrm{p}<0.001 * * \mathrm{p}<0.01 * \mathrm{p}<0.05$

w.r.t. control \#\#\# p <0.001 \# p<0.05 
Table 3: Correlation coefficient ( $r$ ) between hand grip strength and different anthropometric measures of right hand (RH) and left hand (LH) among orthopedically challenged and control male and female subjects

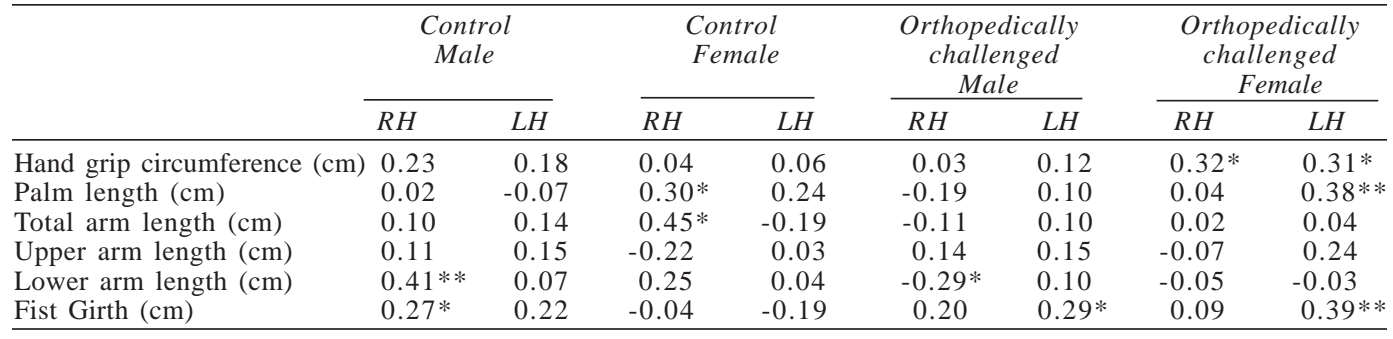

** $\mathrm{p}<0.01 * \mathrm{p}<0.05$

hand grip circumference in both the hands, and with palm length and fist girth in case of left hand. But in case of male orthopedically challenged subjects grip strength had a significant positive correlation with fist girth in the left hand and significant negative correlation with lower arm length in the right hand. It was also been revealed that there was no significant correlation between grip strength and hand grip circumference among control subjects of both sexes. In female subjects the total arm length and palm length of the right hand were significantly correlated with hand grip strength. In case of male subjects a significant correlation was observed between grip strength and lower arm length and fist girth of the right hand.

The orthopedically challenged subjects are divided into three categories according to their source of problems, as mentioned earlier. The significance of difference of grip strength among the subjects of those three groups was evaluated by analysis of variance (ANOVA). Incase of male orthopedically challenged subjects there was no significant difference in hand grip strength among three categories of orthopedically challenged male subjects in both hand (Table 4). On the other hand, in orthopedically challenged female subjects there was a significant difference $(\mathrm{p}<$ 0.01 ) in hand grip strength among three categories of female orthopedically challenged subject in left hand, although no such difference is noted in the right hand.

A post hoc analysis of hand grip strength among different categories of orthopedically

Table 4: One way ANOVA of hand grip strength $(\mathrm{Kg})$ among different category of orthopedically challenged male and female subjects

\begin{tabular}{|c|c|c|c|c|c|}
\hline \multirow[t]{2}{*}{ Sex } & \multirow{2}{*}{$\begin{array}{l}\text { Category of orthopedically } \\
\text { challenged subjects }\end{array}$} & \multicolumn{4}{|c|}{ Grip strength (kg) } \\
\hline & & $R H$ & $F$ ratio & $L H$ & $F$ ratio \\
\hline \multirow{3}{*}{$\begin{array}{l}\text { Orthopedically } \\
\text { challenged Male }\end{array}$} & Stroke case $(n=23)$ & $15.09 \pm 10.08$ & \multirow[t]{3}{*}{1.26} & $18.26 \pm 10.07$ & \multirow[t]{3}{*}{0.76} \\
\hline & Arthritis case $(n=7)$ & $11.57 \pm 5.97$ & & $14.0 \pm 6.06$ & \\
\hline & Accidental case $(n=26)$ & $18.02 \pm 11.10$ & & $19.08 \pm 10.12$ & \\
\hline \multirow{3}{*}{$\begin{array}{l}\text { Orthopedically } \\
\text { challenged Female }\end{array}$} & Stroke case $(n=6)$ & $6.08 \pm 2.76$ & \multirow[t]{3}{*}{2.79} & $14.17 \pm 8.21$ & \multirow[t]{3}{*}{$6.26 * *$} \\
\hline & Arthritis case $(n=26)$ & $11.87 \pm 3.62$ & & $12.48 \pm$ & \\
\hline & Accidental case $(n=27)$ & $10.81 \pm 7.92$ & & $7.32 \pm$ & \\
\hline
\end{tabular}

$* * \mathrm{p}<0.01$

Table 5: Post hoc analysis of grip strength (Kg) of female orthopedically challenged subjects

\begin{tabular}{|c|c|c|c|c|c|c|c|}
\hline \multirow{2}{*}{\multicolumn{2}{|c|}{$\begin{array}{l}\text { Category of orthopedically } \\
\text { challenged subjects } \\
\text { Hand type }\end{array}$}} & \multicolumn{2}{|c|}{ Stroke case } & \multicolumn{2}{|c|}{ Arthritis case } & \multicolumn{2}{|c|}{ Accidental case } \\
\hline & & RH & $L H$ & RH & $L H$ & RH & $L H$ \\
\hline \multirow{3}{*}{$\begin{array}{l}\text { Mean } \\
\text { Stroke case }\end{array}$} & & 6.08 & 14.17 & 11.87 & 12.48 & 10.81 & 7.32 \\
\hline & Mean difference & & & -5.79 & 1.69 & -4.73 & 6.85 \\
\hline & Significant level & & & $\mathrm{P}<0.001$ & NS & $\mathrm{P}<0.01$ & $\mathrm{P}<0.05$ \\
\hline \multirow[t]{2}{*}{ Arthritis case } & Mean difference & & & & & 1.06 & 5.16 \\
\hline & Significant level & & & & & NS & $\mathrm{P}<0.01$ \\
\hline
\end{tabular}


challenged subjects, viz., stroke case, accidental case and arthritis case was made in female subjects (Table 5). From this analysis it was noted that patients with stroke cases had significantly lower grip strength than that of the patients with arthritis in the right hand. However, in case of left hand, the strength of patients with stroke cases showed significantly higher values than that of the patients with accidental cases. The subjects with arthritis cases had significantly higher strength than that of accidental cases in the left hand $(\mathrm{p}<0.01)$.

\section{DISCUSSION}

The magnitude of hand grip strength of orthopedically challenged and control subject showed appreciable difference. Previous studies (Rantanen et al. 1998) have demonstrated that muscle strength differs in disabled elderly women. A remarkably lower grip strength (31\% to $52 \%)$ in orthopedically challenged subjects might be due to the muscle weakness, which was caused by the diseases (arthritis or stroke) or accidents. Häkkinen et al. (2002) suggested that decreased muscle strength of the upper and lower extremities as well as trunk has been repeatedly reported in rheumatoid arthritis patients. The percentage of decrease in strength might depend on the degree of severity of the abnormality. Rice et al. (1998) reported that rheumatoid arthritis caused a $90 \%$ decrease in grip strength compared to healthy persons. In addition to that the physically strong persons usually perform more physical work than the orthopedically challenged persons. The relative disuse of muscles in orthopedically challenged persons might cause atrophy of the same. Other workers (O'Reilly et al. 1997; Kelli et al. 2005) also showed that the patients with osteoarthritis had muscle weakness and possessed lower grip strength than that of normal subjects.

Sex is another important factor which showed a large difference in grip strength among the subjects. Leyk et al. (2007) also found considerable differences in hand-grip strength between young male and female adults. Ranganathan et al. (2001) found a $43 \%$ grip strength decrease in older women compared to older men, vs. $34 \%$ less grip strength in young women when compared to young men. Similar results were also appeared from study of other groups of investigators (Balogum et al. 1991; Richards et al. 1992 and Su et al. 1994). The male subjects of the present study possessed appreciably greater grip strength ( $28 \%$ to $39 \%$ ) than that of the female subjects both in cases of orthppedically challenged and control subjects. The sex difference in hand grip strength was likely due to higher levels of androgenic hormones (Page et al. 2005), greater muscle mass (Kallman et al. 1990) and greater height and weight in boys and men (Kamarul et al. 2006; Kuh et al. 2006). Further, the activity level might be another factor related to the gender difference in grip strength. Usually men perform with greater work load than that of women in professional work as well as in daily activities.

The male subjects had higher anthropometric measures than that of the female subjects. However, all the body dimensions were not significantly different between the orthopedically challenged subjects and control subjects of both the sexes. Therefore, it appeared from the results that all the physical characteristics of subjects not always influenced the grip strength. The grip strength had a poor correlation with different body dimensions in both orthopedically challenged and control subjects.

The difference of grip strength in different category of orthopedically challenged women might be due to the variation in the degree of abnormality. The reasons behind the weakening the muscle was different in three cases. Lower value $(42.91 \%)$ in grip strength in right side of the women having stroke may due to the fact that they have been affected unilaterally as a result of stroke (Boissy et al. 1999). Mc Aniff et al. (2002) suggested that if a person was impaired only on one side, a unilateral task might be possible, whereas a bilateral task might be difficult or impossible. However, only $17.0 \%$ of the population was found to be weak on only one side, which tends to downplay the significance of this limitation. From the post hoc analysis of the present data it has been noted that in stroke case there was a significant difference in hand grip strength when compared to that of the arthritis case and accidental case of female orthopedically challenged subject and in arthritis case there was a significant difference in hand grip strength with accidental case of female orthopedically challenged subjects. Therefore, the hand grip strength may not be differentiating properly in different categories of orthopedically challenged subject. However, lower sample number may be the cause of non-significant difference between 
hand grip strength and different categories of orthopedically challenged subject both in male and female subjects.

\section{CONCLUSION}

The hand grip strength of the orthopedically challenged and normal persons varied as a function of sex. In addition to those different anthropometric measures, especially hand dimensions did not influence the hand grip strength of orthopedically challenged and normal persons. The data of orthopedically challenged persons may be used in designing control panel, switches and other hand arm operated devices for them. It may be designed differentially for different category of orthopedically challenged subjects. The data may be used by the manufacturers who are engaged in making tools and furniture for the orthopedically challenged.

\section{REFERENCES}

Balogun JA, Akomolafe CT, Amusa LO 1991. Grip Strength: Effect of testing posture and elbow position. Arch Phys Med Rehabil, 72(5): 280-3.

Boissy P, Bourbonnais D, Carlotti MM, Gravel D, Arsenault BA 1999. Maximal grip force in chronic stroke subjects and its relationship to global upper extremity function. Clinical Rehabilitation, 13(4): 354-362.

Chaparro A, Rogers M, Fernandez J, Bohan M, Choi SD et al 2000. Range of motion of the wrist: implications for designing computer input devices for the elderly. Disabi Rehabil, 22: 633-637.

Ermakova SV, Podstavkina TP, Strokina AN 1985. Anthropometric Atlas, Recommendation of Methods. New Delhi: Oxonian Press.

Fess EE, Moran C 1981. Clinical Assessment Recommendations. Indianapolis: American Society of Hand Therapists.

Häkkinen A, Hannonen P, Nyman K, Häkkinen K 2002. Aerobic and neuromuscular capacity both in early and long-term rheumatoid arthritis compared to healthy controls. Scand J Rheumatol, 31: 345-50.

Janssen TW, van Oers CA, van der Woude LH, Hollander AP 1994. Physical strain in daily life of wheelchair users with spinal cord injuries. Med Sci Sports Exerc, 26(6): 661-670.

Kallman DA, Plato CC, Tobin JD 1990. The role of muscle loss in the age related decline of grip strength: Cross-sectional and longitudinal perspectives. Journal of Gerontology, 45(3): M82-88.

Kamarul T, Ahmad TS, Loh WYC 2006. Hand grip strength in the adult Malaysian population. Journal of Orthopedic Surgery, 14(2): 172-177.

Kelli LD, Joanne MJ, Jordan BR, Virginia BK 2005. Relationship of radiographic and clinical variables to pinch and grip strength among individuals with osteo arthritis. Arthritis and Rheumatism, 52: 14241430 .
Kuh D, Hardy R, Butterworth S, Okell L, Wadsworth M et al 2006. Developmental origins of midlife grip strength: Findings from a birth cohort study. Journals of Gerontology Series A: Biological Sciences and Medical Sciences, 61: 702-706.

Leyk D, Gorges W, Ridder D, Wunderlich M, Ruther T et al 2007. Hand-grip strength of young men, women and highly trained female athletes. Eur J Appl Physiol, 99: 415-421.

Mathiowetz V, Rennells C, Donahoe L 1985a. Effect of elbow position on grip and key pinch strength. $J$ Hand Surg [AM], 10(5): 694-7.

Mathiowetz V, Kashman N, Volland G, Weber K, Dowe M et al 1985b. Grip and pinch strength: normative data for adults. Arch Phys Med Rehabil, 66: 69-72.

Mc Aniff CM, Bohannon RW 2002. Validity of grip strength dynamometry in acute rehabilitation. $J$ Phys Ther Sci, 14: 41-46.

Mercierand C, Bourbonnais D 2004. Relative shoulder fexor and handgrip strength is related to upper limb function after stroke. Clinical Rehabilitation, 18(2): 215-221.

Muralidhar A, Bishu RR 2000. Safety performance of gloves using the pressure tolerance of the hand. Ergonomics, 43: 561-572.

O'Reilly S, Jones A, Doherty M 1997. Muscle weakness in osteoarthritis. Curr Opin Rheumatol, 9(3): 259262.

Page ST, Amory JK, Bowman FD, Anawalt BD, Matsumoto $\mathrm{AM}$ et al 2005. Exogenous testosterone (T) alone or with finasteride increases physical performance, grip strength, and lean body mass in older men with low serum T. Journal of Clinical Endocrinology \& Metabolism, 90(3): 1502-1510.

Petersen P, Petrick M, Connor H, Conklin D 1989. Grip strength and hand dominance: Challenging the $10 \%$ rule. American Journal of Occupational Therapy, 43(7): $444-447$.

Porter MM, Vandervoort AA and Lexell J 1995. Aging of human muscle: structure, function and adaptability. Scandinavian Journal of Medicine \& Science in Sports, 5(3): 129-142.

Ranganathan VK, Siemionow V, Sahgal V, Yue GH 2001. Effects of aging on hand function. $J$ A Geriatr Soc, 49: $1478-1484$.

Rantanen T, Guralnik JM, Leveille S, Izmirlian G, Hirsch $\mathrm{R}$ et al. 1998. Racial differences in muscle strength in disabled older women. J Gerontol A Biol Sci Med Sci, 53: B355-361.

Rice MS, Leonard C, Carter M 1998. Grip strengths and required forces in accessing everyday containers in a normal population. Am J Occup Ther, 52: 621-626.

Richards L, Olson B, Palmitter-Thomas P 1996. How forearm position affects grip strength. Am J Occup Ther, 50: 133-9.

Rimmer JH 2001. Physical fitness levels of persons with cerebral palsy. Dev Med Child Neurol, 43: 208-212.

Sodhi HS 1991. Sports Anthropometry (A Kinanthropometric Approach). Mohali: ANOVA Publications, pp.176-185.

Su CY, Lin JH, Chien TH, Cheng KF, Sung YT 1994. Grip strength: relationship to shoulder position in normal subjects. Archives Physical and Medical Rehabilitation, 75: 812-815. 
Sunderland A, Tinson D, Bradley L, Hewer R 1989. Arm function after stroke. An evaluation of grip strength as a measure of recovery and a prognostic indicator I Neurol Neurosurg Psychiatry, 52(11): 1267-72.

Weiner JS, Lourie JA 1969. Human Biology, A Guide to Field Method. Oxford: Blackwell Scientific Publications.
Young A 1986. Exercise physiology in geriatric practice. Acta Med Scand Suppl, 711: 227-232.

Zhang Y, Niu1 J, Kelly-Hayes M, Chaisson CE, Aliabadi $\mathrm{P}$ et al. 2002: Prevalence of Symptomatic Hand Osteoarthritis and Its Impact on Functional Status among the Elderly-The Framingham Study. American Journal of Epidemiology, 156(11): 1021-1027. 